\title{
On Variable-Scale Piecewise Stationary Spectral Analysis of Speech Signals for ASR ${ }^{\star}$
}

\author{
Vivek Tyagi ${ }^{\mathrm{a}, \mathrm{c}, *}$ Hervé Bourlard ${ }^{\mathrm{b}, \mathrm{c}}$ Christian Wellekens ${ }^{\mathrm{a}, \mathrm{c}}$ \\ a Institute Eurecom, P.O Box: 193, Sophia-Antipolis, France. \\ ${ }^{\mathrm{b}}$ IDIAP Research Institute,P.O Box: 592, Martigny, Switzerland. \\ ${ }^{\mathrm{c}}$ Swiss Federal Institute of Technology, Lausanne, Switzerland.
}

\begin{abstract}
It is often acknowledged that speech signals contain short-term and long-term temporal properties [15] that are difficult to capture and model by using the usual fixed scale (typically 20ms) short time spectral analysis used in hidden Markov models (HMMs), based on piecewise stationarity and state conditional independence assumptions of acoustic vectors. For example, vowels are typically quasi-stationary over $40-80 \mathrm{~ms}$ segments, while plosive typically require analysis below $20 \mathrm{~ms}$ segments. Thus, fixed scale analysis is clearly sub-optimal for "optimal" time-frequency resolution and modeling of different stationary phones found in the speech signal. In the present paper, we investigate the potential advantages of using variable size analysis windows towards improving state-of-the-art speech recognition systems. Based on the usual assumption that the speech signal can be modeled by a timevarying autoregressive (AR) Gaussian process, we estimate the largest piecewise quasi-stationary speech segments, based on the likelihood that a segment was generated by the same AR process. This likelihood is estimated from the Linear Prediction (LP) residual error. Each of these quasi-stationary segments is then used as an analysis window from which spectral features are extracted. Such an approach thus results in a variable scale time spectral analysis, adaptively estimating the largest possible analysis window size such that the signal remains quasi-stationary, thus the best temporal/frequency resolution tradeoff. The speech recognition experiments on the OGI Numbers95 database[19], show that the proposed variable-scale piecewise stationary spectral analysis based features indeed yield improved recognition accuracy in clean conditions, compared to features based on minimum cross entropy spectrum [1] as well as those based on fixed scale spectral analysis.
\end{abstract}

Key words: variable-scale quasi-stationary analysis, speech spectral analysis 


\section{Introduction}

Most of the Automatic Speech Recognition (ASR) acoustic features, such as Mel-Frequency Cepstral Coefficient (MFCC)[16] or Perceptual Linear Prediction (PLP)[17], are based on some sort of representation of the smoothed spectral envelope, usually estimated over fixed analysis windows of typically $20 \mathrm{~ms}$ to $30 \mathrm{~ms}$ of the speech signal $[16,15]$. Such analysis is based on the assumption that the speech signal can be assumed to be quasi-stationary over these segment durations. Typically, the vowels last for 40 to $400 \mathrm{~ms}$ while the stops last for 3 to $250 \mathrm{~ms}$. However, the sustained-stationary segments in a vowel can typically last from 30 to $80 \mathrm{~ms}$, while stops are time-limited by less than 20ms [15]. Therefore, it implies that the spectral analysis based on a fixed size window of $20 \mathrm{~ms}$ or $30 \mathrm{~ms}$ has some limitations, including:

- The frequency resolution obtained for quasi-stationary segments (QSS) longer than 20 or $30 \mathrm{~ms}$ is quite low compared to what could be obtained using larger analysis windows. Although, most of the frequency resolution is lost due to averaging by 24 Mel filters. However, power spectrum estimation (DFT) over quasi-stationary segments will still lead to low-variance Melfilter bank energies as compared to those obtained with a fixed scale spectral analysis that does not take quasi-stationarity into account.

- In certain cases, the analysis window can span the transition between two QSSs, thus blurring the spectral properties of the QSSs, as well as of the transitions. Indeed, in theory, Power Spectral Density (PSD) cannot even be defined for such non stationary segments [9]. Furthermore, on a more practical note, the feature vectors extracted from such transition segments do not belong to a single unique (stationary) class and may lead to poor discrimination in a pattern recognition problem.

In this work, we make the usual assumption that the piecewise quasi-stationary segments (QSS) of the speech signal can be modeled by a Gaussian AR process of a fixed order $p$ as in $[2,4,10,11]$. We then formulate the problem of detecting QSSs as a Maximum Likelihood (ML) detection problem, defining a QSSs as the longest segment that has most probably been generated by the same AR process. ${ }^{1}$ As is well known, given a $p^{\text {th }}$ order AR Gaussian QSS, the Minimum Mean Square Error (MMSE) linear prediction (LP) filter parameters $[a(1), a(2), \ldots a(p)]$ are the most "compact" representation of that QSS amongst

\footnotetext{
* This work was supported by European Commission's 6th Framework Program project, DIVINES under the contract number FP6-002034.

* Corresponding author: Vivek Tyagi

Email addresses: tyagi@eurecom.fr, bourlard@idiap.ch, welleken@eurecom.fr (Christian Wellekens).

1 Equivalent to the detection of the transition point between the two adjoining QSSs.
} 
all the $p^{\text {th }}$ order all pole filters [9]. In other words, the normalized "coding error" 2 is minimum amongst all the $p^{\text {th }}$ order LP filters. When erroneously analyzing two distinct $p^{\text {th }}$ order AR Gaussian QSSs in the same non-stationary analysis window, it can be shown that the "coding error" will then always be greater than the ones resulting of QSSs analyzed individually in stationary windows[14]. This is intuitively satisfying since, in the former case, we are trying to encode ' $2 p^{\prime}$ free parameters (the LP filter coefficients of each of the QSS) using only $p$ parameters (as the two distinct QSS are now analyzed within the same window). Therefore, higher coding error is expected in the former case as compared to the optimal case when each QSS is analyzed in a stationary window. As further explained in the next sections, this forms the basis of our criteria to detect piecewise quasi-stationary segments. Once the "start" and the "end" points of a QSS are known, all the speech samples coming from this QSS are analyzed within that window, resulting in (variablescale) acoustic vectors.

Working under the similar framework, Brandt [10] had proposed a maximum likelihood algorithm for speech segmentation. However, there are certain subtle theoretical as well practical differences in the proposed approach and the Brandt's algorithm which are described in Section 3. Brandt's approach was again followed in [11], where the authors proposed several speech segmentation algorithms. However, none of these papers[10,11] attempted to perform stationary spectral analysis as has been done in this paper. Using a parametric model that the speech signal is generated by a time-varying auto-regressive process, we have shown the relationship between ML segmentation and piecewise stationary spectral analysis in Section 4. Although, there has been plenty of research on speech signal segmentation (including speaker change detection), quite limited work has been done to interlink signal segmentation and quasi-stationary spectral analysis as has been done in this work.

In [11], the author has illustrated certain speech waveforms with segmentation boundaries overlaid. The validity of their algorithm is shown by a segmentation experiment, which on an average, segments phonemes into 2.2 segments. This result is quite useful as a pre-processor for the manual transcription of speech signals. However, the author in [11] did not discuss or extend the ML segmentation algorithm as a variable-scale quasi-stationary spectral analysis technique suitable for ASR, as done in the present work.

In [3], Atal has described a temporal decomposition technique, with applications in speech coding, to represent the continuous variation of the LPC parameters as a linearly weighted sum of a number of discrete elementary components. These elementary components are designed such that they have

$\overline{2}$ The power of the residual signal normalized by the number of samples in the window 
the minimum temporal spread (highly localized in time) resulting in superior coding efficiency. However, the relationship between the optimization criterion of "the minimum temporal spread" and the quasi-stationarity is not obvious. Therefore, the discrete elementary components are not necessarily quasi-stationary and vice-versa.

Coifman et al [6] have described a minimum entropy basis selection algorithm to achieve the minimum information cost of a signal relative to the designed orthonormal basis. In [8], Srinivasan et. al. have proposed a multi-scale QSS technique for noisy speech enhancement which is based on Coifman's technique [6]. In [4], Svendsen et al have proposed a ML segmentation algorithm using a single fixed window size for speech analysis, followed by a clustering of the frames which were spectrally similar for sub-word unit design. We emphasize here that this is different from the approach proposed here where we use variable size windows to achieve the objective of piecewise quasi-stationary spectral analysis. More recently, Achan et al [13] have proposed a segmental HMM for speech waveforms which identifies waveform samples at the boundaries between glottal pulse periods with applications in pitch estimation and time-scale modifications.

Our emphasis in this paper is on better spectral modeling of the speech signal rather than achieving better coding efficiency or reduced information cost. Nevertheless, we believe that these two objectives are somewhat fundamentally related. The main contribution of the present paper is to demonstrate that the variable-scale QSS spectral analysis technique can possibly improve the ASR performance as compared to the fixed scale spectrum analysis. Moreover, we show the relationship between the maximum likelihood QSS detection algorithm and the well known spectral matching property of the LP error measure [5]. Finally, we do a comparative study of the proposed variablescale spectrum based features and the minimum cross-entropy time-frequency distributions developed by Loughlin et al [1].

In the sequel of this paper, Section 2 formulates the ML detection problem for identifying the transition points between QSS. Section 3 compares the proposed approach with Brandt's[10] approach. In Section 4, we illustrate an analogy of the proposed technique with spectral matching property of the LP error measure. Finally, the experimental setup and results are described in Section 5 . 


\section{ML Detection of the change-point in an AR Gaussian random process}

Consider an instance of a $p^{\text {th }}$ order AR Gaussian process, $\mathbf{x}[\mathbf{n}], n \in[1, N]$ whose generative LP filter parameters can either be $\mathbf{A}_{0}=\left[1, a_{0}(1), a_{0}(2) \ldots a_{0}(p)\right]$ or can change from $\mathbf{A}_{1}=\left[1, a_{1}(1), a_{1}(2) \ldots a_{1}(p)\right]$ to $\mathbf{A}_{2}=\left[1, a_{2}(1), a_{2}(2) \ldots a_{2}(p)\right]$ at time $n_{1}$ where $n_{1} \in[1, N]$. As usual, the excitation signal is assumed to be drawn from a white Gaussian process and its power can change from $\sigma=\sigma_{1}$ to $\sigma=\sigma_{2}$. The general form of the Power Spectral Density (PSD) of this signal is then known to be

$$
P_{x x}(f)=\frac{\sigma^{2}}{\left|1-\sum_{i=1}^{p} a(i) \exp (-j 2 \pi i f)\right|^{2}}
$$

where $a(i)$ s are the LPC parameters. The hypothesis test consists of:

- $\mathbf{H}_{0}$ : No change in the PSD of the signal $x(n)$ over all $n \in[1, N]$, LP filter parameters are $\mathbf{A}_{0}$ and the excitation (residual) signal power is $\sigma_{0}$.

- $\mathbf{H}_{1}$ : Change in the PSD of the signal $x(n)$ at $n_{1}$, where $n_{1} \in[1, N]$, LP filter parameters change from $\mathbf{A}_{1}$ to $\mathbf{A}_{2}$ and the excitation(residual) signal power changes from $\sigma_{1}$ to $\sigma_{2}$.

Let, $\hat{\mathbf{A}}_{0}$ denote the maximum likelihood estimate (MLE) of the LP filter parameters and $\hat{\sigma}_{0}$ denote the MLE of the residual signal power under the hypothesis $\mathbf{H}_{0}$. The MLE estimate of the filter parameters is equal to their MMSE estimate due to the Gaussian distribution assumption [2] and, hence, can be computed using the Levinson Durbin algorithm [9] without significant computational cost.

Let $\mathbf{x}_{1}$ denote $\left[x(1), x(2), \ldots x\left(n_{1}\right)\right]$ and $\mathbf{x}_{2}$ denote $\left[x\left(n_{1}+1\right), \ldots x(N)\right]$. Under hypothesis $\mathbf{H}_{1},\left(\hat{\mathbf{A}}_{1}, \hat{\sigma}_{1}\right)$ are the MLE of $\left(\mathbf{A}_{1}, \sigma_{1}\right)$ estimated on $\mathbf{x}_{1}$, and $\left(\hat{\mathbf{A}}_{2}\right.$, $\left.\hat{\sigma}_{2}\right)$ are the MLE of $\left(\mathbf{A}_{2}, \sigma_{2}\right)$ estimated on $\mathbf{x}_{2}$, where $\mathbf{x}_{1}$ and $\mathbf{x}_{2}$ have been assumed to be independent of each other. A Generalized Likelihood Ratio Test (GLRT) [14] would then pick hypothesis $\mathbf{H}_{1}$ if

$$
\log L(\mathbf{x})=\log \left(\frac{p\left(\mathbf{x}_{1} \mid \hat{\mathbf{A}}_{1}, \hat{\sigma}_{1}\right) p\left(\mathbf{x}_{2} \mid \hat{\mathbf{A}}_{2}, \hat{\sigma}_{2}\right)}{p\left(\mathbf{x} \mid \hat{\mathbf{A}}_{0}, \hat{\sigma}_{0}\right)}\right)>\gamma
$$

where $\gamma$ is a decision threshold that will have to be tuned on some development set. Given that the total number of samples in $\mathbf{x}_{1}$ and $\mathbf{x}_{2}$ is the same as in $\mathbf{x}_{0}$, their likelihoods can be compared directly in (2). Under the hypothesis $\mathbf{H}_{0}$ the entire segment $\mathbf{x}=[x(1) \ldots x(N)]$ is considered stationary and the MLE $\hat{\mathbf{A}}_{0}$ is computed via the Levinson-Durbin algorithm using all the samples in segment $\mathbf{x}$. It can be shown that the MLE $\hat{\sigma}_{0}$ is the power of the residual signal $[2,14]$. Under $\mathbf{H}_{1}$, we assume that there are two distinct QSS, namely $\mathbf{x}_{1}$ and $\mathbf{x}_{2}$. 
The MLE $\hat{\mathbf{A}}_{1}$ and $\hat{\mathbf{A}}_{2}$ are computed via the Levinson-Durbin algorithm using samples from their corresponding QSS. MLE $\hat{\sigma}_{1}$ and $\hat{\sigma}_{2}$ are computed as the power of the corresponding residual signals. In fact, $p\left(\mathbf{x} \mid \hat{\mathbf{A}}_{0}, \hat{\sigma}_{0}\right)$ is equal to the probability of residual signal obtained using the filter parameters $\hat{\mathbf{A}}_{0}$, yielding:

$$
p\left(\mathbf{x} \mid \hat{\mathbf{A}}_{0}, \hat{\sigma}_{0}\right)=\frac{1}{\left(2 \pi \hat{\sigma}_{0}^{2}\right)^{N / 2}} \exp \left[\frac{-1}{2 \hat{\sigma}_{0}^{2}} \sum_{n=1}^{N}\left(e_{0}^{2}(n)\right)\right]
$$

where $e_{0}(n)$ is the residual error and

$$
e_{0}(n)=x(n)-\sum_{i=1}^{p} a_{0}(i) x(n-i), \quad n \in[1, N]
$$

and

$$
\hat{\sigma}_{0}^{2}=\frac{1}{N} \sum_{n=1}^{N} e_{0}^{2}(n)
$$

Similarly, $p\left(\mathbf{x}_{1} \mid \hat{\mathbf{A}}_{1}, \hat{\sigma}_{1}\right)$ and $p\left(\mathbf{x}_{2} \mid \hat{\mathbf{A}}_{2}, \hat{\sigma}_{2}\right)$ are the likelihoods of the residual signal vectors of the $A R$ models $\mathbf{A}_{1}$ and $\mathbf{A}_{2}$, respectively, and have the same functional forms as above. Substituting these expressions into (2) yields

$$
\log L(\mathbf{x})=\frac{1}{2} \log \left[\frac{\hat{\sigma}_{0}^{N}}{\hat{\sigma}_{1}^{n_{1}} \hat{\sigma}_{2}^{\left(N-n_{1}\right)}}\right]
$$

In the present form, the GLRT $\log L(\mathbf{x})$ has now a natural interpretation. Indeed, if there is a transition point in the segment $\mathbf{x}$ then it has, in effect, $2 p$ degrees of freedom. Under hypothesis $\mathbf{H}_{0}$, we encode $\mathbf{x}$ using only $p$ degrees of freedom (LP parameters $\hat{\mathbf{A}}_{0}$ ) and, therefore, the coding (residual) error $\hat{\sigma}_{0}^{2}$ will be high. However, under hypothesis $\mathbf{H}_{1}$, we use $2 p$ degrees of freedom (LP parameters $\hat{\mathbf{A}}_{1}$ and $\hat{\mathbf{A}}_{2}$ ) to encode $\mathbf{x}$. Therefore, the coding (residual) errors $\hat{\sigma}_{1}^{2}$ and $\hat{\sigma}_{2}^{2}$ can be minimized to reach the lowest possible value. ${ }^{3}$ This will result in $L(\mathbf{x})>1$. On the other hand, if there is no AR switching point in the segment $\mathbf{x}$ then it can be shown that, for large $n_{1}$ and $N$, the coding errors are all equal $\left(\hat{\sigma}_{0}^{2}=\hat{\sigma}_{1}^{2}=\hat{\sigma}_{2}^{2}\right)$. This will result in $L(\mathbf{x}) \simeq 1$.

An interesting and useful property of the GLRT in (4) is that it is invariant to any multplicative scale factor of signal $\mathbf{x}$. For example let us consider a scaled segment $\mathbf{y}=c \times \mathbf{x}$, where $\mathrm{c}$ is a constant. As the LP filter and the inverse LP filter are both linear filters, a scaled input signal will result in an output signal with the same multiplicative scale factor. Therefore, the residual signals

3 When $\hat{\mathbf{A}}^{1}$ and $\hat{\mathbf{A}}^{2}$ are estimated, strictly based on the samples from the corresponding quasi-stationary segments. 
obtained after analyzing $\mathbf{y}$ will be $e_{0}^{y}(n)=c \times e_{0}(n), e_{1}^{y}(n)=c \times e_{1}(n), e_{2}^{y}(n)=$ $c \times e_{2}(n) \forall n \in[1, N]$. Therefore the GLRT in (4) will become,

$$
\begin{aligned}
\log L(\mathbf{y}) & =\frac{1}{2} \log \left[\frac{c^{N} \hat{\sigma}_{0}^{N}}{c^{n_{1}} \hat{\sigma}_{1}^{n_{1}} c^{N-n_{1}} \hat{\sigma}_{2}^{\left(N-n_{1}\right)}}\right] \\
& =\log L(\mathbf{x})
\end{aligned}
$$

This ensures that even if the speech signal might have varying power levels (different scale factors) GLRT in (5) can still be compared to a fixed threshold $\gamma$. However, if there are abrupt energy changes within a segment, then the GLRT will most likely classify them as different QSSs.

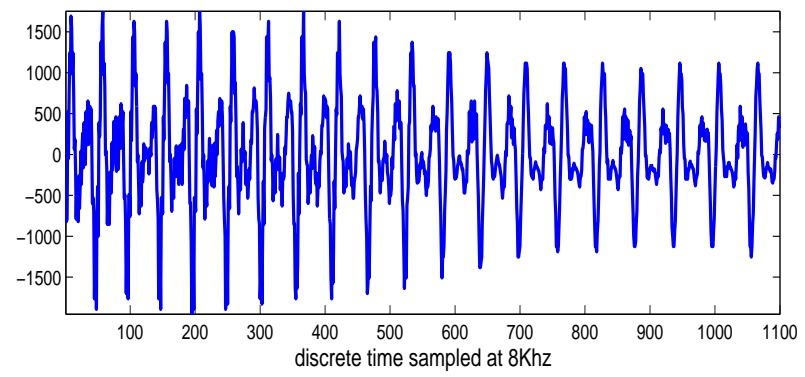

Plot of GLRT

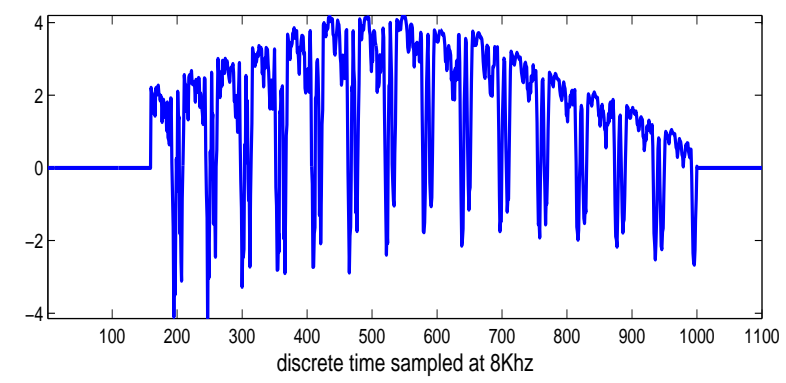

Fig. 1. Typical plot of the Generalized log likelihood ratio test (GLRT) for a voiced speech segment. The sharp downward spikes in the GLRT are due to the presence of a glottal pulse at the beginning of the right analysis window $\left(\boldsymbol{x}_{2}\right)$. The GLRT peaks around the sample 500 which marks as a strong AR model switching point

An example is illustrated in Fig. 1. The top pane shows a segment of a voiced speech signal. In the bottom figure, we plot the GLRT as the function of the hypothesized change over point $n$. Whenever, the right window i.e the segment $\mathbf{x}_{2}$ spans the glottal pulse in the beginning of the window, the GLRT exhibits strong downward spikes (negative values of the GLRT), which is due to the fact that the LP filter cannot predict large samples occurring in the beginning of the window. However, these negative spikes of the GLRT do not affect our decision as we are comparing GLRT to a large positive threshold (typically 3.5). Therefore, in a way, we are comparing only the positive envelope of the GLRT to a pre-selected positive threshold. Consequently, the sharp negative spikes in GLRT caused due to the occurrence of a glottal pulse in right win- 


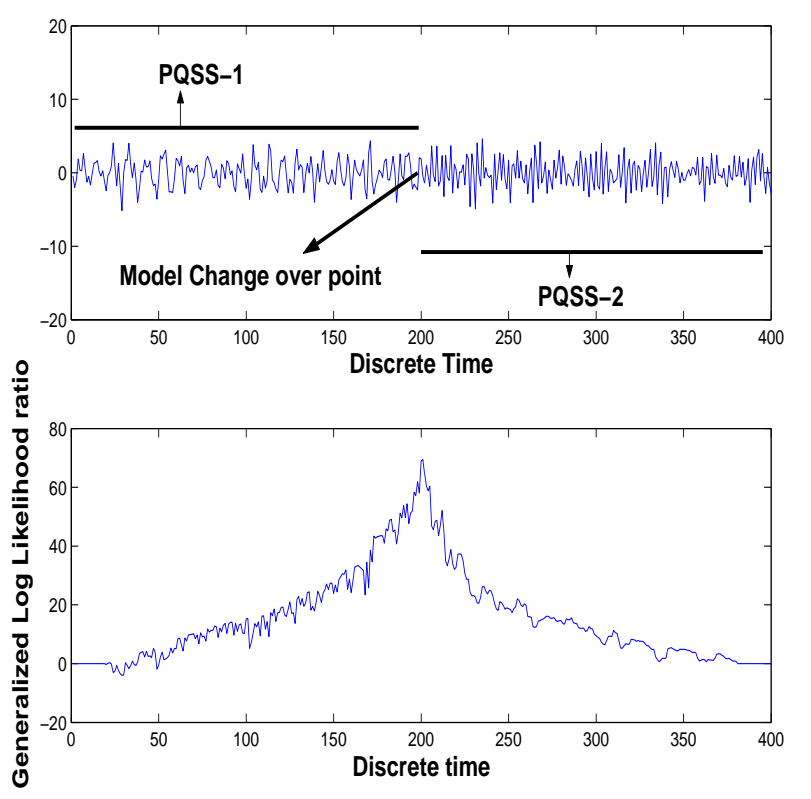

Fig. 2. Typical plot of the Generalized log likelihood ratio test (GLRT) for an unvoiced speech segment that consists of two piece-wise quasi-stationary segments (PQSS). The GLRT peaks around the sample 200 which is indeed an AR model switching point.

dow i.e $\mathbf{x}_{2}$ will bring down the GLRT value, thus preventing the GLRT from exceeding a positive threshold. This has a desirable effect that as long as the pitch periods are nearly similar(stationary voiced segment), they will never be segmented into different QSSs. Instead, they will be glued together to form one QSS. The minimum sizes of the left and the right windows are 160 and 100 samples respectively and the reasons for this choice are explained in Section 5 . This also explains the zero value of the GLRT at the beginning and the end of the whole test segment. The GLRT peaks around sample 500 which marks a strong AR model switching point. In Fig. 2, we plot the GLRT of an unvoiced speech segment that consists of two QSSs. As can be seen from the Fig. 2, in the case of the unvoiced speech, the GLRT has a rather smooth envelope due to the absence of the glottal pulses. The GLRT peaks around sample 200 that marks an AR model switching point. The algorithm presented in this paper does not make any distinction between voiced and unvoiced speech segments. GLRT of all the segments in an utterance are compared to a fixed threshold that has been tuned on a development set. This results in a sequence of speech segments that are usually of variable lengths. We note that the segments returned by the algorithm are quasi-stationary only in a probabilistic sense that the event that two adjacent segments are instances of the same stationary process is $e^{- \text {Threshold }}$ times as likely as the event that they are instances of two different stationary processes. Therefore the choice of the threshold decides the trade-off between false acceptance and false rejection of QSSs. However, as we are primarily interested in improved recognition accuracies, we have tuned the threshold on a development set based on the recognition accuracies. 


\section{Comparison with Brandt's algorithm}

The GLRTs in both Brandt's approach and the proposed approach are the same as in (4). This is not surprising as in both the approaches GLRT is a maximum likelihood solution under the assumption that the speech signal is a realization of a Gaussian AR process where the AR parameters can change over time. However, the differences lie in the methods employed to estimate the residual powers $\sigma_{0}, \sigma_{1}, \sigma_{2}$. In our approach, the AR parameters $\mathbf{A}_{0}, \mathbf{A}_{1}, \mathbf{A}_{2}$ and the residual powers $\sigma_{0}, \sigma_{1}, \sigma_{2}$. are estimated by solving the least squares equations [9] over their corresponding segments, $\mathbf{x}_{0}, \mathbf{x}_{1}, \mathbf{x}_{2}$ respectively. To solve these least squares equations, we use the so-called autocorrelation method [9] (page:486) that leads to an autocorrelation matrix that is Toeplitz. Toeplitz matrices can be inverted quite efficiently using the Levinson Durbin algorithm[9](pages:254). Therefore the computational overload of our approach is quite low.

Whereas, Brandt used the so-called covariance method [9](page:486) to solve for the AR parameters and the residual powers, $\sigma_{0}, \sigma_{1}, \sigma_{2}$.. This method leads to an autocorrelation matrix that is non-Toeplitz, thus excluding the use of fast Levinson-Durbin algorithm. Brandt has used the lattice-filters[9](pages:280) to estimate the AR parameters and the residual powers. Lattice filters are also quite efficient as compared to the Gram-Schmidt orthogonalization, but less so as compared to Levinson-Durbin algorithm. Therefore, the proposed approach is faster than Brandt's approach. Use of the autocorrelation method [9] guarantees a minimum phase all-pole filter[9]. However, the covariance method [9] does not necessarily lead to a minimum phase all-pole filter. Therefore, the proposed approach ensures a stable all-pole filter as opposed to Brandt's approach.

In Brandt's algorithm, the left window i.e $\mathbf{x}_{1}$ uses a growing memory covariance ladder algorithm and the right window $\mathbf{x}_{2}$ uses a sliding memory covariance ladder algorithm. Initialization of the growing memory covariance ladder algorithm requires certain intermediate quantities that are provided by the sliding memory covariance ladder algorithm which operates on $\mathbf{x}_{2}$. Hence, the AR parameters $\mathbf{A}_{1}$ and the residual power $\sigma_{1}$ are indirectly influenced by the samples in the right window $\mathbf{x}_{2}$. To compensate for this, Brandt's algorithm uses a second search called "jump time optimization process" to estimate the stationarity change-over point.

Whereas, in our approach the AR parameters $\mathbf{A}_{1}, \mathbf{A}_{2}$ and the residuals $\sigma_{1}, \sigma_{2}$ are estimated using samples strictly from their corresponding segments i.e. $\mathbf{x}_{1}, \mathbf{x}_{2}$ respectively. Therefore, in the proposed approach there is just one step stationarity change point detection. Whenever the GLRT in (4) exceeds the threshold $\gamma$, a stationarity change point is recorded. This is in contrast to 
Brandt's method where this step is followed by a "jump time optimization process" which, finally estimates the stationarity change point.

\section{Relation of GLRT to Spectral Matching}

In the section will show the relationship between the maximum likelihood segmentation and the spectral matching which is one of the main contributions of this paper. As is well known the LP error measure possesses the spectral matching property [5]. Specifically, given a speech segment $\mathbf{x}$, let its power spectrum (periodogram) be denoted by $\mathbf{X}\left(e^{j \omega}\right)$. Let the all pole model spectrum of the segment $\mathbf{x}$ be denoted as $\hat{\mathbf{X}}_{0}\left(e^{j \omega}\right)$. Then it can be shown that the MMSE error $\sigma_{0}^{2}$ of the LP filter estimated over the entire segment $\mathbf{x}$ is given by $[5]$.

$$
\begin{aligned}
\sigma_{0}^{2} & =\int_{-\pi}^{\pi} \frac{\mathbf{X}\left(e^{j \omega}\right)}{\hat{\mathbf{X}}_{0}\left(e^{j \omega}\right)} d \omega \text { where } \\
\hat{\mathbf{X}}_{0}\left(e^{j \omega}\right) & =\frac{1}{\left|1-\sum_{i=1}^{p} a_{0}(i) \exp (-j 2 \pi i f)\right|^{2}}
\end{aligned}
$$

Therefore minimizing the residual error $\sigma_{0}^{2}$ is equivalent to the minimization of the integrated ratio of the signal power spectrum $\mathbf{X}\left(e^{j \omega}\right)$ to its approximation $\hat{\mathbf{X}}_{0}\left(e^{j \omega}\right)[5]$. Substituting (6) in (4) we obtain,

$$
\log L(\mathbf{x})=\frac{1}{2} \log \frac{\left(\int_{-\pi}^{\pi} \frac{\mathbf{X}\left(e^{j \omega}\right)}{\hat{\mathbf{X}}_{0}\left(e^{j \omega}\right)} d \omega\right)^{N}}{\left(\int_{-\pi}^{\pi} \frac{\mathbf{X}_{1}\left(e^{j \omega}\right)}{\hat{\mathbf{X}}_{1}\left(e^{j \omega}\right)} d \omega\right)^{n_{1}}\left(\int_{-\pi}^{\pi} \frac{\mathbf{X}_{2}\left(e^{j \omega}\right)}{\hat{\mathbf{X}}_{2}\left(e^{j \omega}\right)} d \omega\right)^{N-n_{1}}}
$$

where, $\mathbf{X}\left(e^{j \omega}\right), \mathbf{X}_{1}\left(e^{j \omega}\right)$ and $\mathbf{X}_{2}\left(e^{j \omega}\right)$ are the power spectra of the segments $\mathbf{x}$, $\mathbf{x}_{1}$ and $\mathbf{x}_{2}$ respectively. Similarly $\hat{\mathbf{X}}_{0}\left(e^{j \omega}\right), \hat{\mathbf{X}}_{1}\left(e^{j \omega}\right)$ and $\hat{\mathbf{X}}_{2}\left(e^{j \omega}\right)$ are the MMSE $p^{\text {th }}$ order all-pole model spectra estimated over the segments $\mathbf{x}, \mathbf{x}_{1}$ and $\mathbf{x}_{2}$ respectively. Therefore, $\hat{\mathbf{X}}_{0}\left(e^{j \omega}\right), \hat{\mathbf{X}}_{1}\left(e^{j \omega}\right)$ and $\hat{\mathbf{X}}_{2}\left(e^{j \omega}\right)$ are the best LP spectral matches to their corresponding power spectra. One way of interpreting (8) is that it is a measure of the relative goodness between the best LP spectral match achieved by modeling $\mathbf{x}$ as a single QSS and the best LP spectral matches obtained by assuming $\mathbf{x}$ to consist of two distinct QSS, namely $\mathbf{x}_{1}$ and $\mathbf{x}_{2}$. This is further explained as follows. If $\mathbf{x}_{1}$ and $\mathbf{x}_{2}$ are indeed two distinct QSS, then $\mathbf{X}_{1}\left(e^{j \omega}\right)$ and $\mathbf{X}_{2}\left(e^{j \omega}\right)$ will be quite different and $\mathbf{X}\left(e^{j \omega}\right)$ will be a gross average of these two spectra. In other words, the frequency support of $\mathbf{X}\left(e^{j \omega}\right)$ will be a union of those of the $\mathbf{X}_{1}\left(e^{j \omega}\right)$ and $\mathbf{X}_{2}\left(e^{j \omega}\right) . \hat{\mathbf{X}}_{1}\left(e^{j \omega}\right)$ and $\hat{\mathbf{X}}_{2}\left(e^{j \omega}\right)$, having $p$ poles each, will match their corresponding power spectra reasonably well, resulting in a lower value of the denominator in (8). However, 
$\hat{\mathbf{X}}_{0}\left(e^{j \omega}\right)$ will be a relatively poorer spectral match to $\mathbf{X}\left(e^{j \omega}\right)$ as it has only $p$ poles to account for the wider frequency support. Therefore we incur a higher spectral mismatch by assuming $\mathbf{x}$ to be a single QSS when in fact it is composed of two distinct QSS $\mathbf{x}_{1}$ and $\mathbf{x}_{2}$. This results in the GLRT $\log L(\mathbf{x})$ taking up a high value. Whereas if $\mathbf{x}_{1}$ and $\mathbf{x}_{2}$ are the instances of the same quasi-stationary process, then so is $\mathbf{x}$. Therefore $\mathbf{X}_{1}\left(e^{j \omega}\right), \mathbf{X}_{2}\left(e^{j \omega}\right)$ and $\mathbf{X}\left(e^{j \omega}\right)$ are nearly the same with similar all-pole models, resulting in a value of the GLRT close to zero. The above discussion points to the fact that the QSS analysis based on the proposed GLRT is constantly striving to achieve a better time varying spectral modeling of the underlying signal as compared to single fixed scale spectral analysis. However, the above discussion is true only if the speech signal can be assumed to have been generated from a Gaussian AR process of a fixed order $p$, where the AR parameters can change over time. This limitation arises from the fact that one needs to assume a signal generative model based on which one can develop a criterion of stationarity.

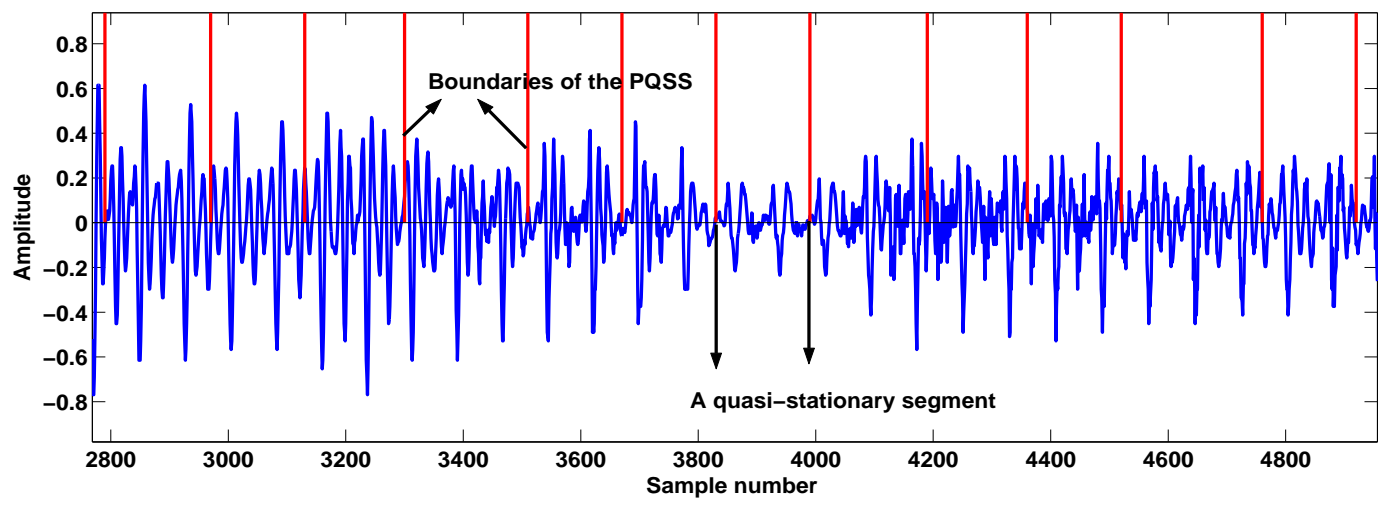

Fig. 3. Quasi stationary segments (QSS) of a speech signal as detected by the algorithm with $\gamma=3.5$ and LP order $p=14$.

\section{$5 \quad$ Experiments and Results}

We have used the GLRT L(x) in (4) to perform QSS spectral analysis of speech signals for ASR applications. We initialize the algorithm with a left window size $\mathrm{W} \_\mathrm{L}=20 \mathrm{~ms}$ and a right window size $\mathrm{W} \_\mathrm{R}=12.5 \mathrm{~ms}$. We compute their corresponding MMSE residuals and the MMSE residual of the union of the two windows using the Levinson-Durbin algorithm. Then, the GLRT is computed using (4) and is compared to the threshold. The choice of the threshold $\gamma=3.5$ was decided on the basis of ASR results on a development set. In figure (3), we illustrate the boundaries of the QSS as detected by the algorithm with $\gamma=3.5$. In general, the ASR results are slightly sensitive to the threshold, although not in a huge way. If the GLRT is greater than the threshold $\gamma$, W_L is considered the largest possible QSS and we obtain a spectral estimate using all the samples in W_L. Otherwise, $W_{\_} \mathrm{L}$ is incremented by INCR $=1.25 \mathrm{~ms}$ and 
the whole process is repeated until GLRT exceeds $\gamma$ or W_L becomes equal to the maximum window size WMAX $=60 \mathrm{~ms}$. The computation of a MFCC feature vector from a very small segment (such as 10ms) is inherently very noisy. ${ }^{4}$ Therefore, the minimum duration of a QSS as detected by the algorithm was constrained to be $20 \mathrm{~ms}$. Ideally the right window size $W \_R$ should be as small as possible so that we can instantaneously detect a stationarity change point. However, a reliable estimate of the AR parameters and the corresponding residual signal requires sufficiently large number of samples in the analysis window. Therefore as a compromise between these two opposing factors, we have chosen the $\mathrm{W} R=12.5 \mathrm{~ms}$. Throughout the experiments, a fixed LP order $p=14$ was used.

The likelihood ratio test is quite widely used for speaker segmentation [12] where the average length of a single speaker segment may last from 1sec to several seconds. This provides a relatively large amount of samples to estimate the parameters of the probability density functions as compared to the present problem where we have to first estimate the generative AR parameters and the corresponding residuals to detect stationarity change over point within $20 \mathrm{~ms}$ to $60 \mathrm{~ms}$. Moreover, in speaker change detection one can use the aprioriinformation that a speaker will at least speak for a second or so. Therefore most of the time it can be safely assumed that there will not be more than two speakers within one second long speech segment. Hence, a local maxima of the GLRT within a time-span of one second can be used as a speaker-change point. This approach has been successfully used in [12]. However, in our case the QSSs can have much more variable durations ranging from $3 \mathrm{~ms}$ to $80 \mathrm{~ms}^{5}$. Therefore, there is no minimum duration in which we can assume that only two QSSs will be present, thus excluding the use of local maxima of the GLRT as an estimate of the stationarity change-over point.

Before proceeding further, however, we feel necessary to briefly discuss certain inconsistencies between variable-scale spectral analysis and state-of-the-art Hidden Markov models ASR using Gaussian mixture models (HMM-GMM). HMM-GMM systems typically use spectral features based on a constant window size (typically $20 \mathrm{~ms}$ ) and a constant shift size (typically $10 \mathrm{~ms}$ ). The shift size determines the Nyquist frequency of the cepstral modulation spectrum [7], which is typically measured by the delta features of the static MFCC or PLP features. In a variable-scale piecewise quasi-stationary analysis, the shift size should preferably be equal to the size of the detected QSS. Otherwise, if the shift size is $\mathrm{x} \%$ of the duration of the QSS, then the next detected QSS will be

\footnotetext{
${ }^{4}$ Due to very few DFT samples falling under the the Mel-filter bins resulting in high variance of the mel-filter bank energies

5 However, as we require sufficiently large number of samples to reasonably estimate the AR parameters and the residuals to compute the GLRT, the proposed algorithm can only detect QSSs larger than and equal to $20 \mathrm{~ms}$
} 
the same but of duration $(100-x) \%$ and the following one will be of duration $(100-2 x) \%$ and so on until we have shifted past the entire duration of the QSS. This results in the undesirable effect that the same QSS gets analyzed by successively smaller windows, hence increasing the variance of the feature vector of this QSS. On the other hand, the use of a shift size equal to the variable window size will change the Nyquist frequency of the cepstral modulation spectrum [7]. Therefore, the modulation frequency pass-band of the delta filters [7] will vary from frame to frame and may suffer from aliasing for shift sizes in excess of $20 \mathrm{~ms}$.

To avoid fluctuating Nyquist frequency of the cepstral modulation spectrum[7], a fixed shift size of $12.5 \mathrm{~ms}$ was used in the algorithm. As explained above, this sometimes resulted in the undesirable effect that the same QSS gets analyzed by progressively smaller windows. To alleviate this problem, the zeroth cepstral coefficient $c(0)$, which is a non-linear function of the windowed signal energy and, hence, of the window size, was normalized such that its dependence on the window size is minimized.

In order to assess the effectiveness of the proposed algorithm, speech recognition experiments were conducted on the OGI Numbers corpus [19]. This database contains spontaneously spoken free-format connected numbers over a telephone channel. The lexicon consists of 31 words. Figure (4) illustrates the distribution of the QSSs as detected by the proposed algorithm. Nearly $47 \%$ segments were analyzed with the smallest window size of $20 \mathrm{~ms}$ and they mostly corresponded to short-time limited segments. However, voiced segments and long silences were mostly analyzed by using longer windows in the range $30 \mathrm{~ms}-60 \mathrm{~ms}$. The short peak at $60 \mathrm{~ms}$ is due to the accumulated value over all the segments that should have been longer than $60 \mathrm{~ms}$ but were constrained by our choice of the largest window size.

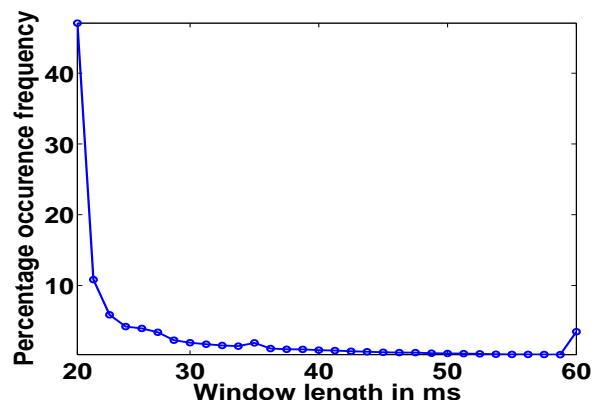

Fig. 4. Distribution of the QSS window sizes detected and then used in the training set

Throughout the experiments, MFCC coefficients and their temporal derivatives were used as speech features. However, five feature sets were compared. In [1], Loughlin et al proposed using a geometric mean of multiple spectrograms of different window sizes to overcome the time-frequency limitation of 
any single spectrogram. They showed that combining the information content from multiple spectrograms in form of their geometric mean, is optimal for minimizing the cross entropy between the multiple spectra. We have followed their approach to derive MFCC features (noted as Minimum cross-entropy MFCC) from the geometric mean of the multiple power spectra computed over varying window sizes, specifically $20 \mathrm{~ms}, 30 \mathrm{~ms}, 40 \mathrm{~ms}$ and $50 \mathrm{~ms}$.

(1) [39 dim. MFCC:] computed over a fixed window of length $20 \mathrm{~ms}$.

(2) [39 dim. MFCC:] computed over a fixed window of length $50 \mathrm{~ms}$.

(3) [78 dim. Concatenated MFCC: ] a concatenation of the above two feature vectors.

(4) [Minimum cross entropy,39 dim MFCC:] MFCC computed from the geometric mean of the power spectra computed from $20 \mathrm{~ms}, 30 \mathrm{~ms}, 40 \mathrm{~ms}$ and $50 \mathrm{~ms}$ long windows.

(5) [Variable-scale QSS MFCC+Deltas:] For a given frame, the window size is dynamically chosen using the proposed algorithm ensuring that the windowed segment is quasi-stationary.

Hidden Markov Model and Gaussian Mixture Model (HMM-GMM) based speech recognition systems were trained using public domain software HTK [18] on the clean training set from the original Numbers corpus. The speech recognition results in clean conditions for various spectral analysis techniques are given in table 1. The fixed scale MFCC features using $20 \mathrm{~ms}$ and $50 \mathrm{~ms}$ long analysis windows have $5.8 \%$ and $5.9 \%$ word error rate (WER) respectively. The concatenation of MFCC feature vectors derived from $20 \mathrm{~ms}$ and $50 \mathrm{~ms}$ long windows has a $5.7 \%$ WER and it has twice the number of HMM-GMM parameters as compared to the rest of the systems ${ }^{6}$. The slight improvement in this case may be due to the multiple scale information present in this feature, albeit in an ad-hoc way. The minimum cross-entropy MFCC features which were derived from the geometric mean of the power spectra computed over $20 \mathrm{~ms}, 30 \mathrm{~ms}$, $40 \mathrm{~ms}$ and $50 \mathrm{~ms}$ long analysis windows, have a WER of $5.7 \%$. The proposed variable-scale system which adaptively chooses a window size in the range $[20 \mathrm{~ms}, 60 \mathrm{~ms}]$, followed by the usual MFCC computation, has a $5.0 \%$ WER. This corresponds to a relative improvement of nearly $10 \%$ over the rest of the techniques

\section{Conclusion}

We have demonstrated that the variable-scale piecewise quasi-stationary spectral analysis of speech signal can possibly improve the state-of-the-art ASR. Such a technique can partially overcome the time-frequency resolution lim-

$\overline{6}$ Due to twice the feature dimension as compared to the rest of the systems 
Table 1

Word error rate in clean conditions

\begin{tabular}{|l|c|}
\hline MFCC $20 \mathrm{~ms}$ & 5.8 \\
\hline MFCC $50 \mathrm{~ms}$ & 5.9 \\
\hline Concat. MFCC $(20 \mathrm{~ms}, 50 \mathrm{~ms})$ & 5.7 \\
\hline Min. Cross entropy based MFCC & 5.7 \\
\hline Proposed Variable-scale QSS MFCC & 5.0 \\
\hline
\end{tabular}

itations of the fixed scale spectral analysis techniques. However, it can be argued that most of the frequency resolution is anyway lost due to Mel-filter binning of the DFT samples. Nevertheless, a spectrum(DFT) estimated over a quasi-stationary segment will help to reduce the variance of the estimated Mel-filter bank energies and consequently those of the MFCC feature vectors. However, as we need certain minimum number of samples to estimate the AR parameters and the residuals, our algorithm cannot detect QSSs below 20ms. Comparisons were drawn with the other competing multi-scale techniques such as the minimum cross-entropy spectrum. The proposed technique led to the minimum WER as compared to the rest of the techniques. Although, the performance gains are modest, we believe that further work on variable scale quasi-stationary analysis can overcome the limitations of a fixed scale spectral analysis of speech signal leading to better ASR performances.

\section{References}

[1] P. Loughlin, J. Pitton and B. Hannaford, "Approximating Time-Frequency Density Functions via Optimal Combinations of Spectrograms," IEEE Signal Processing Letters, vol.1, No.12, December 1994.

[2] F. Itakura, " Minimum Prediction Residual Principle Applied to Speech Recognition," IEEE Trans. on ASSP, Vol.23, no.1, February 1975.

[3] B. S. Atal, "Efficient coding of LPC parameters by temporal decomposition," In the Proc. of IEEE ICASSP, Boston, USA, 1983.

[4] T. Svendsen, K. K. Paliwal, E. Harborg, P. O. Husoy, "An improved sub-word based speech recognizer," Proc. of IEEE ICASSP, 1989.

[5] J. Makhoul, "Linear Prediction: A Tutorial Review, " In the Proc. of IEEE, vol.63, No.4, April 1975.

[6] R. R. Coifman and M. V. Wickerhauser, " Entropy based algorithms for best basis selection, " IEEE Trans. on Information Theory, Vol.38, Issue:2, March 1992. 
[7] V. Tyagi, I McCowan, H. Bourlard, H. Misra, "Mel-Cepstrum Modulation Spectrum (MCMS) features for Robust ASR, " In the Proc. of IEEE ASRU 2003, St. Thomas, Virgin Islands, USA.

[8] S. Srinivasan and W. B. Kleijn, "Speech Enhancement Using Adaptive timedomain Segmentation," In the Proc. of ICSLP 2004, Jeju, S. Korea.

[9] S. Haykin, Adaptive Filter Theory, Prentice-Hall Publishers, N.J., USA, 1993.

[10] A. V. Brandt, "Detecting and estimating the parameters jumps using ladder algorithms and likelihood ratio test," in Proc. of ICASSP, Boston, MA, 1983,pp. 1017-1020.

[11] R. A. Obrecht, "A new Statistical Approach for the Automatic Segmentation of Continuous Speech Signals, " IEEE Trans. on ASSP, vol.36, No.1, January 1988.

[12] J. Ajmera, I. McCowan and H. Boulard, "Robust Speaker Change Detection," IEEE Signal Processing Letters, vol.11, No. 8, August 2004.

[13] K. Achan, S. Roweis, A. Hertzmann and B. Frey, "A Segmental HMM for Speech Waveforms, " UTML Techical Report 2004-001, Dept. of Computer Science, Univ. of Toronto, May 2004.

[14] S. M. Kay, Fundamentals of Statistical Signal Processing: Detection Theory, Prentice-Hall Publishers, N.J., USA, 1998.

[15] L. Rabiner and B. H. Juang, Fundamentals of Speech Recognition, PrenticeHall, N.J., USA, 1993.

[16] S. B. Davis and P. Mermelstein, "Comparison of Parametric Representation for Monosyllabic Word Recognition in Continuously Spoken Sentences, "IEEE Trans. on ASSP, Vol. ASSP-28, No. 4, August 1980.

[17] H. Hermansky, "Perceptual linear predictive (PLP) analysis of speech, " J. Acoust. Soc. Am., vol.87:4, April 1990.

[18] S. Young, J. Odell, D. Ollason, V. Valtchev, and P. Woodland, The HTK Book, Cambridge University, 1995.

[19] R. A. Cole, M. Fanty, and T. Lander, "Telephone speech corpus at CSLU," Proc. of ICSLP, Yokohama, Japan, 1994. 